\title{
LOADING RATE EFFECT ON THE DOUBLE-K FRACTURE PARAMETERS OF CONCRETE
}

\author{
GONZALO RUIZ ${ }^{* \dagger}$, JOSÉ J. ORTEGA ${ }^{\dagger}$, RENA C. YU ${ }^{\dagger}$, SHILANG XU ${ }^{\dagger \dagger}$ AND YAO WU ${ }^{\dagger \dagger}$ \\ ${ }^{\dagger}$ Universidad de Castilla-La Mancha \\ Ciudad Real, Spain \\ ${ }^{\dagger \dagger}$ Zhejiang University \\ Hangzhou, China \\ *e-mail: Gonzalo.Ruiz@uclm.es \\ JoseJoaquin.Ortega@uclm.es \\ Chengxiang.Yu@uclm.es \\ SIXu@zju.edu.cn \\ WuYao19870508@163.com
}

Key words: Double- $K$ method, Rate effect, Elastic equivalence.

\begin{abstract}
This work studies the effect of the loading rate on the double- $K$ fracture parameters in concrete. These are the crack initiation toughness $K_{I c}^{\text {ini }}$ and the unstable toughness $K_{I c}^{\text {un }}$, which mark the two main stages of crack propagation and are calculated from a load-crack mouth opening curve, $P-w_{M}$. However, the original methodology does not take into account the influence of the load velocity, which may not be negligible for certain testing configurations adopted to avoid an excessive test duration. The double- $K$ method includes a third parameter, the cohesive toughness $K_{I c}^{c}$, that relates $K_{I c}^{i n i}$ and $K_{I c}^{u n}$ by integrating the cohesive stresses in the fracture process zone. In this paper, the influence of the loading rate is introduced in the method through $K_{I c}^{c}$ by including in the softening law a viscous factor, function of the crack opening velocity. The idea arises from the viscous-cohesive approach implemented in a numerical model successfully used in a previous work (Engineering Fracture Mechanics 82:195-208, 2012). Rate-dependent double- $K$ parameters are computed from $P-w_{M}$ curves obtained with the numerical model, analysing a wide range of specimens and crack opening rates. $R$-curves are also obtained. The results show that double- $K$ parameters increase with the loading rate. The rate dependence is slightly greater for large specimens, which amplifies the size effect.
\end{abstract}

\section{INTRODUCTION}

The double- $K$ method is a calculation methodology for concrete and other quasibrittle materials that obtains as fracture parameters two values of toughness: $K_{I c}^{i n i}$, which marks the initation of the crack propagation, and $K_{I c}^{u n}$, which indicates the limit at which the crack propagation becomes unstable. Double- $K$ parameters and methodology were introduced by Profs.
Reinhardt and $\mathrm{Xu}$ in the late 90's [1-4] and have generated abundant scientific literature since then. They are fairly used by many researchers and practitioners around the world, especially in China, where there is already a standard to regulate the determination of the double- $K$ parameters [5].

This method is based in an elastic equivalence, so that analytical formulas from linear elastic fracture mechanics (LEFM) can be applied to calculate those fracture 
parameters. Thus, this methodology stands as a practical tool to determine when a crack would start to grow and how far the structural element is from failure. The elastic equivalence is established for a curve of loadCMOD (crack mouth opening displacement or $w_{M}$ ). Therefore, the application of this method needs the measurement of this curve in laboratory with specimens of the studied concrete. These specimens can be beams for three-point bending (TPB) tests or compact specimens for wedge-splitting tests. The present work has analysed the results for the first type.

Double- $K$ parameters are determined from two points in the $P-w_{M}$ curve. $K_{I c}^{u n}$ needs the maximum load $P^{\max }$ and its corresponding critical crack mouth opening $\left(w_{M c}\right) . K_{I C}^{\text {ini }}$ requires only the load at crack initiation $\left(P^{i n i}\right)$. However, this load is usually difficult to detect in experimental tests, which may require techniques such as the use of strain gauges near the notch tip. There is not a standard procedure to place these strain gauges, which is left to the researcher's personal point of view, resulting in a source of variability for a same case of study. Furthermore, the crack may not develop homogenously throughout the specimen width, starting at different moments on each side, which makes the determination of $P^{i n i}$ still more difficult. Due to these problems, the authors of the method $[2,4]$ proposed a methodology where $K_{I c}^{i n i}$ is obtained from $K_{I c}^{\text {un }}$ by detracting the amount of toughness developed along the fracture process zone (FPZ) from the crack initiation to the peak load, computed by the cohesive toughness $K_{I c}^{c}$.

With this parameter, only $P^{\max }$ and $w_{M c}$ are necessary, which greatly simplifies the required work in the laboratory and data analysis. However, one problem that arises from using $K_{I c}^{c}$ as it was originally defined is that it only deals with data from static tests. Experience has demonstrated the influence of loading velocity in tests results, which may result relevant for many test configurations used in laboratories, with loading rates that are not quasi-static or when unstable portions appear in $P-w_{M}$ curves, when the control of the machine cannot follow fast variations of the load. Besides, rate-effects couple with the ones deriving from the size of the specimen [6].

The main objective of this study is, then, to adapt the double- $K$ methodology so that the loading velocity influence can be estimated, whether it is necessary to correct rate-affected double- $K$ parameters or static input data is wanted to be used for extrapolating to different loading rates.

The introduction of rate-effects in the double- $K$ methodology is made following the approach of Rosa et al. [7], who developed a viscous-cohesive model which was implemented in the so-called 'smeared cracktip superposition scheme' to obtain an exact cohesive solution. It gives the cohesive stresses as a function of both the crack opening and the crack opening velocity. The same viscous-cohesive model is now introduced in the double- $K$ methodology by means of a viscous factor implemented in the cohesive toughness $K_{I c}^{c}$. This parameter allows computing rate-dependent toughness values and deriving the corresponding $R$ curves.

This numerical model was calibrated for a certain concrete characterised by previous experimental tests. With it, $P-w_{M}$ curves were obtained, both static and rate-affected, in order to apply the double- $K$ method to them. The values of $K_{I C}^{c}$ obtained from each group of $P$ $w_{M}$ curves are compared to observe their relation. Moreover, different calculations with the rate-affected $K_{I c}^{c}$ are performed to analyse the particular effects of considering different crack opening rates or viscous parameters.

The results show that the toughness parameters increase with the loading velocity. The rate dependence is slightly weaker for small specimens than for large ones. This amplifies the size effect on the double- $K$ parameters, which is pronounced for small sizes and mild for big sizes at strictly static tests.

The article is structured as follows: Section 2 presents the experimental programme and its results; in Section 3, the numerical model is described and new $P-w_{M}$ curves are obtained; in Section 4, the double- $K$ method is analysed and modified to include the loading rate influence; in Section 5, the results are 
discussed; finally, Section 6 presents the conclusions of the work.

\section{EXPERIMENTAL PROGRAMME}

Concrete characterisation tests were used for calibrating the numerical model for a certain material and geometry. The experimental programme consisted in TPB tests, using notched beams. The tests were done with crack opening control, registering load $(P)$ and crack mouth opening displacement $\left(w_{M}\right)$ values.

The geometry of the beams consisted in prisms with a span 4 times the depth or size $(D)$, with a central notch whose length is $0.5 \mathrm{D}$. Three different beam sizes were tested: $D 1=75 \mathrm{~mm}, D 2=150 \mathrm{~mm}$ and $D 3=300 \mathrm{~mm}$, keeping the width $(B)$ in $50 \mathrm{~mm}$.

The concrete used was a micro-concrete, with a maximum aggregate size smaller than in usual concretes ( $5 \mathrm{~mm}$ in this case). This option was chosen because the objective is to represent the behaviour of real beams using specimens at a smaller scale. It was intended to obtain a similar $D / l_{c h}$ relation, and microconcretes are usually more brittle, which also means that their characteristic length $l_{c h}$ is smaller.

The material was made under an intense control of manufacture, so that scatter was minimized in the measured values of the mechanical properties. It was characterised by standard tests. The following table shows the mean value for each parameter, with their standard deviation in brackets:

Table 1: Concrete properties.

\begin{tabular}{llll}
\hline$f_{c}(\mathrm{MPa})$ & $f_{t}(\mathrm{MPa})$ & $E_{c}(\mathrm{GPa})$ & $G_{F}(\mathrm{~N} / \mathrm{m})$ \\
\hline $39.5(1.6)$ & $3.8(0.34)$ & $30.5(2.0)$ & $62.5(4.7)$ \\
\hline
\end{tabular}

With these parameters, the resulting characteristic length is $l_{c h}=130 \mathrm{~mm}$.

Each beam size was tested at a different CMOD rate to study the size effect and at a very slow velocity, so that the testing conditions were quasi-static. The displacement rates were scaled in the same proportion than the beam sizes: $D 1,5 \mu \mathrm{m} / \mathrm{min} ; \quad D 2$, $10 \mu \mathrm{m} / \mathrm{min} ; D 3,20 \mu \mathrm{m} / \mathrm{min}$.
In order to avoid an additional consumption of energy during the fracture process, the testing equipment was provided with antitorsion supports, which assure that the applied load does not generate any torsion whatsoever and thus, the crack is only subjected to pure mode I opening. Indeed, in every test the crack grew straight from the notch without being twisted due to unwanted torsional moments.

The experimental results are shown in Fig. 1. These test results are representative as we obtain very similar curves within each beam size.

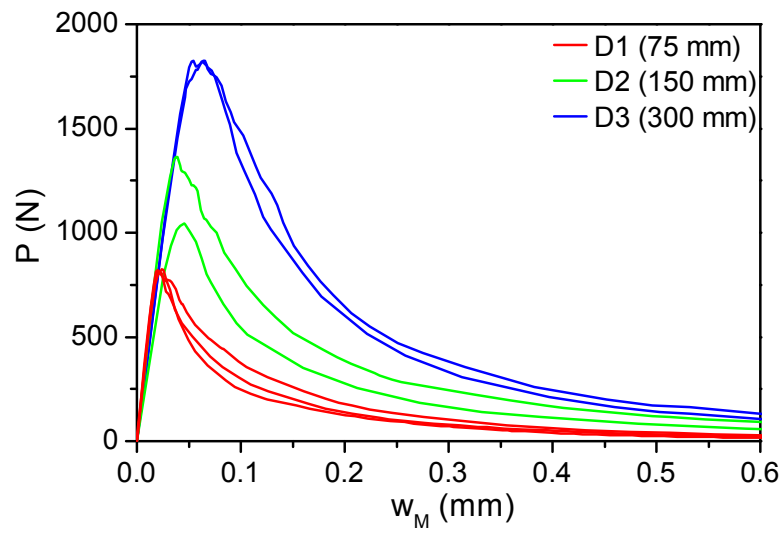

Figure 1: Experimental $P-w_{M}$ curves.

\section{NUMERICAL TESTS}

In order to extend the study beyond the limitations in dimensions of laboratory tests, a numerical model of a standard TPB test was used. This model is validated for this concrete and these specimen dimensions with the previous test results.

The model is based on the smeared crack tip method of Planas and Elices [8], which considers that only one discrete crack develops, in cohesive fracture. The numerical formulation consists in an improvement of the influence method of Petersson [9] by a triangulation of the system of equations, based on the applicability of LEFM and the superposition hypothesis. This system can be solved by direct substitution, making easier the calculation process.

It is necessary to set a cohesive law to relate the stresses in the crack with the corresponding crack openings, $\quad \sigma=f(w)$. Several different softening laws were considered, selecting the one that fitted the test 
results the best. This was the bilinear softening law proposed by Guinea, Planas and Elices [10] (Fig. 2). It is designed to provide a good fitting of the peak load and the tail of the $P-w_{M}$ curve, as demonstrated by the comparison with the experimental curves (Fig. 3). A detailed description of the method and a successful application case is offered in [11], where the numerical model includes a reinforcement layer.

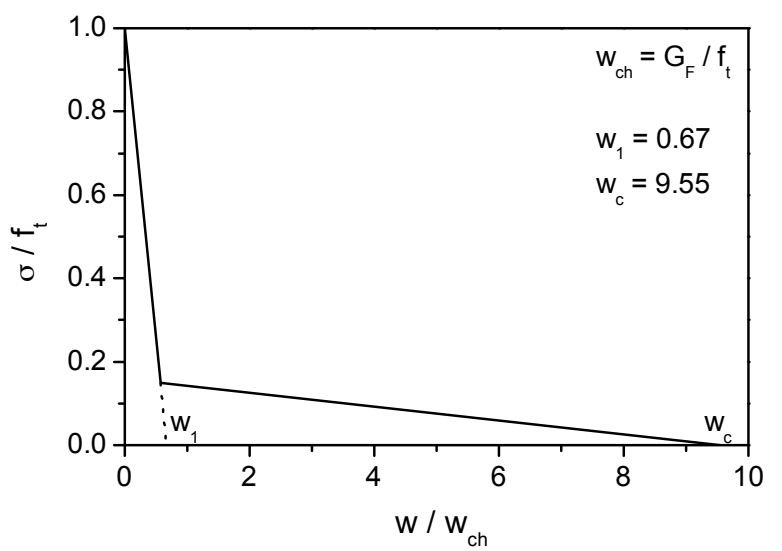

Figure 2: Softening law.

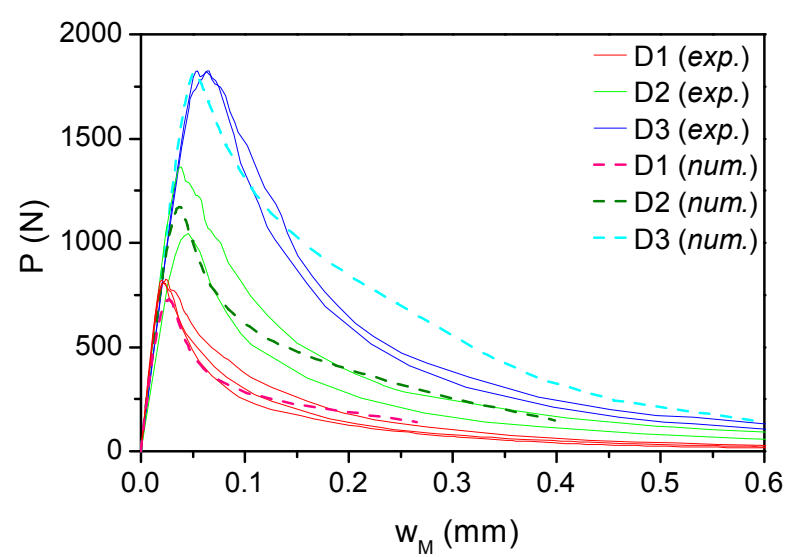

Figure 3: Numerical model validation.

Furthermore, the scope of the model was amplified to include the effect of the loading velocity [7]. High loading rates find higher opposition to break the material due to inertial phenomena. Thus, a simple approach to insert this effect in the model consists in considering a time dependent or viscous factor that multiplies the cohesive stress given by the softening law in the FPZ. This factor has been defined as:

$$
g(\dot{w})=1+\left(\frac{\dot{w}}{\dot{w}_{0}}\right)^{n}
$$

where:

$-\dot{W}$ is the crack opening rate, which includes the time dependence,

- $\dot{w}_{0}$ is a reference crack opening rate and

$-n$ is a constant.

Previous experiences [7] have proved that a good value for $\dot{w}_{0}$ is $100 \mu \mathrm{m} / \mathrm{s}$, while $n$ can be chosen from the range $(0.15,0.30)$.

In this work, a total of 100 nodes have been used in the beam depth, with the notch tip in node 50. In order to validate the model, numerical results are compared with the experimental ones for each of the three sizes studied in laboratory. As the tests were performed under quasi-static conditions, the numerical results have been obtained without including the rate effect. The comparison between experimental and numerical results, shown in Fig. 3, proves the good agreement between them and validates the model for this concrete and geometry.

Once validated, the model is ready to generate the $P-w_{M}$ curves of the size range of interest for this study. This range is established in relation to the characteristic length $\left(l_{c h}\right)$ of the concrete, setting $D / l_{c h}=1$ as the unit value. The chosen range includes the 9 decimal fractions and the 9 multiples until 10 of that reference value. As $l_{c h}=130 \mathrm{~mm}$, this means that the range of sizes that we introduce in the numerical model covers a 19 -size series from $13 \mathrm{~mm}$ to $1300 \mathrm{~mm}$. Figure 4 shows these results.

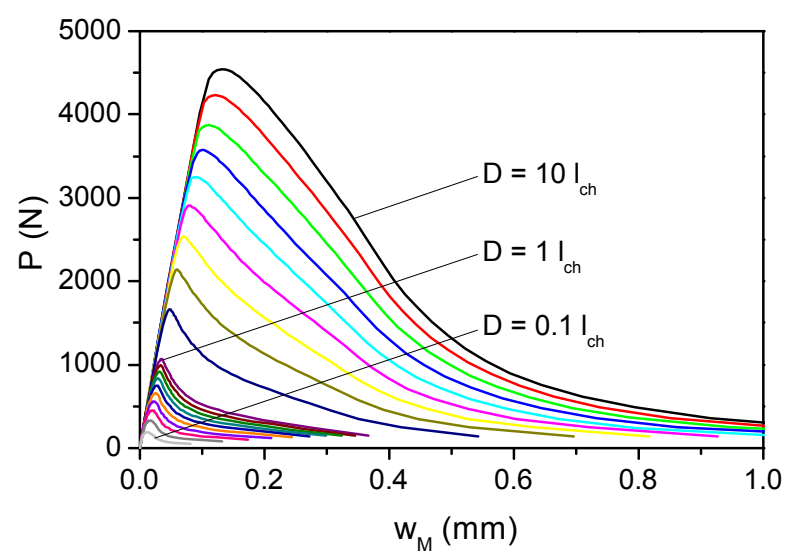

Figure 4: Static numerical $P-w_{M}$ curves. 
Additionally, six more $P-w_{M}$ curves were obtained including the rate influence for sizes from $D=1 l_{c h}(130 \mathrm{~mm})$ to $D=10 l_{c h}$ $(1300 \mathrm{~mm})$. The configuration parameters were: $\quad \dot{w}_{M}=2.5 \mu \mathrm{m} / \mathrm{s}, \quad \dot{w}_{0}=100 \mu \mathrm{m} / \mathrm{s}$, $\mathrm{n}=0.15$. Double- $K$ parameters obtained from these curves are compared with the ones calculated from the static curves. Figure 5 shows three examples of the new curves in comparison with their corresponding static curves.

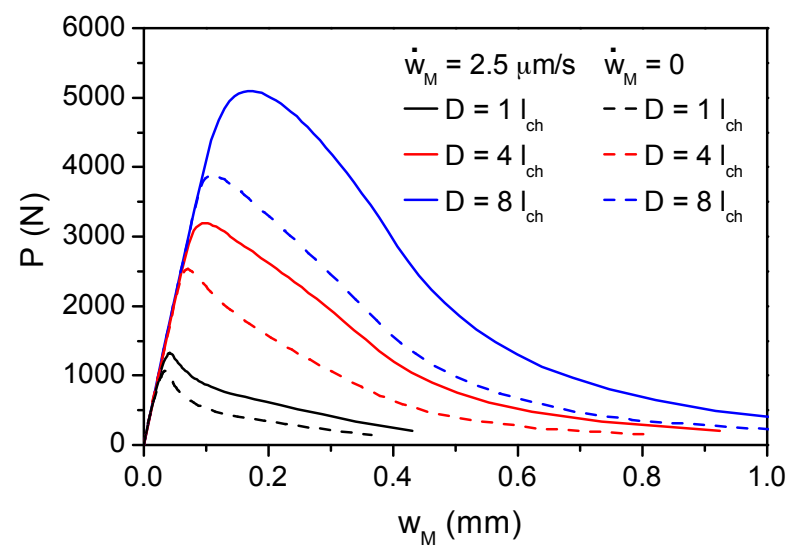

Figure 5: Rate-affected numerical $P-w_{M}$ curves.

\section{RATE INFLUENCE IN THE DOUBLE-K METHOD}

The double- $K$ method obtains two toughness values that mark two corresponding different stages of the fracture process: the crack initiation, defined by $K_{I c}^{i n i}$, and the limit of the stable crack propagation, defined by the unstable toughness $K_{I c}^{\text {un }}$. The toughness is calculated with the formula for the stress intensity factor $K$ (Eq. 2) at a critical situation where the crack is propagating. For the crack opening mode I, the toughness parameter is denoted as $K_{I c}$. It is required to know the equivalent elastic crack length $a$, which can be calculated by Eq. 3. This formula relates each couple of $P-w_{M}$ values with their corresponding crack length. Eq. 2 and Eq. 3 correspond to TPB specimens.

$$
\begin{gathered}
\mathrm{K}_{I}=\frac{3 P S}{2 D^{2} B} \sqrt{a} F_{I}(a / D) \\
w_{M}=\frac{6 P S a}{D^{2} B E} V_{I}(a / D)
\end{gathered}
$$

where:
- $S$ is the span length,

- $D$ is the beam size,

- $B$ is the beam width,

- $E$ is the elastic modulus and

- $F_{I}$ and $V_{I}$ are shape functions for TPB specimens (see [12]).

$K_{I c}^{u n}$ is calculated with the maximum load $P^{\max }$ and its corresponding critical crack mouth opening $w_{M c}$. $K_{I c}^{\text {ini }}$ corresponds to the crack initiation load $P^{i n i}$, with a crack length equal to the initial notch length $a_{0}$.

In order to avoid the problems around the detection of $P^{i n i}$, the cohesion toughness $K_{I C}^{c}$ was introduced $[2,4]$, which computes the toughness developed along the FPZ from the crack initiation $\left(a_{0}\right)$ to the peak load $\left(a_{c}\right)$. Then, $K_{I c}^{i n i}$ can be obtained through Eq. 4:

$$
K_{I c}^{i n i}=K_{I c}^{u n}-K_{I c}^{c}
$$

The calculation of this parameter requires to set a stress distribution, given by a softening law. $K_{I c}^{c}$ is calculated with Eq. 5:

$$
K_{I c}^{c}=\int_{a_{0}}^{a_{c}} \frac{2 \sigma\left(x / a_{c}\right)}{\sqrt{\pi a_{c}}} F\left(\frac{x}{a_{c}}, \frac{a_{c}}{D}\right) d x
$$

where:

- $\sigma\left(x / a_{c}\right)=\sigma\left(w\left[x / a_{c}\right]\right)$, the softening law and

- $F\left(x / a_{c}, a_{c} / D\right)$ is a geometric function.

This integration must be solved by a numerical method. In order to avoid this inconvenience, a simplified method was proposed later [4]. However, in a previous work [13] about the size effect in the double- $K$ parameters, it was found out that the simplified method overestimates the value of $K_{I c}^{c}$ for large specimens, so the integration method is recommended. It was also studied the influence of the cohesive law adopted, which may accentuate or attenuate the size effect, so it is necessary to adopt the proper softening law with fitted parameters for the particular concrete under study.

This method has been applied to the set of $19 P-w_{M}$ static curves obtained from the numerical model. Figure 6 shows the values of the different toughness parameters for each beam size, linked by continuous curves. For $K_{I c}^{i n i}$ there are two different curves. One of them corresponds to the crack initiation load $P^{i n i}$ that can be measured directly from the $P$ - 
$w_{M}$ curve, marked by the loss of linearity in the loading ramp. The other one corresponds to the $K_{I c}^{i n i}$ calculated by Eq. 4. As it is observed, there is a good agreement between them along this wide range of specimen sizes, which backs the usefulness of $K_{I c}^{c}$.

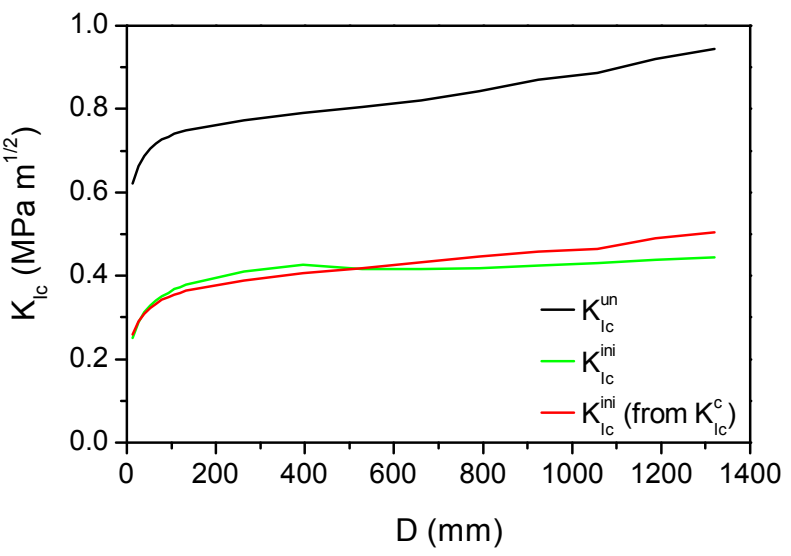

Figure 6: Double- $K$ parameters from static $P-w_{M}$ curves.

After this general description of the double$K$ method, the simplest idea to implement the loading rate influence is by following the same procedure that in the numerical model. There, the softening law is transformed by introducing a viscous factor (Eq. 1) that amplifies the cohesive stress for a given crack opening. Here, $K_{I c}^{c}$ allows the introduction of this same viscous factor as it contains a cohesive law as well. Therefore, the original softening law $\sigma(w)$ in Eq. 5 is substituted by $\sigma(w, \dot{w})$ :

$$
\sigma(w, \dot{w})=f(w) \mathrm{g}(\dot{w})
$$

where:

- $f(w)$ is the softening law, dependent on the crack opening $w$ and

$-g(\dot{w})$ is the viscous factor defined by Eq. 1 .

In the double- $K$ method, an expression developed by Reinhardt et al. [14] was proposed as the softening law. However, any cohesive function adapted to the concrete under analysis and that properly represents its behaviour can be chosen. In this work, the bilinear softening law of Guinea, Planas and Elices [10] was adopted, as this is the one implemented in the numerical model that has been used for generating the $P-w_{M}$ curves taken as input data, with the same parameters.
$K_{I c}^{c}$ is calculated by integrating the cohesive stresses along the crack, from the notch tip $\left(a_{0}\right)$ to the critical crack length $\left(a_{c}\right)$. The stress depends on the position along the crack as the crack opening $w$ varies from the crack opening at the notch tip $\left(w_{T}\right)$ to 0 (at the crack tip), expressed as $w(x)$. Similarly, the crack opening rate $\dot{w}$ depends on the variation of crack opening in a time step, $\dot{w}(w(x), t)$. This is taken into account by recording the crack width in the previous point to the maximum load in the curve so that it is possible to obtain the local opening velocity along the crack until $a_{c}$ (see Fig. 7).

$$
\begin{aligned}
& \Delta t=\frac{W_{M B}-W_{M A}}{\dot{W}_{M}} \\
& \dot{W}(x)=\frac{\Delta W(x)}{\Delta t}
\end{aligned}
$$

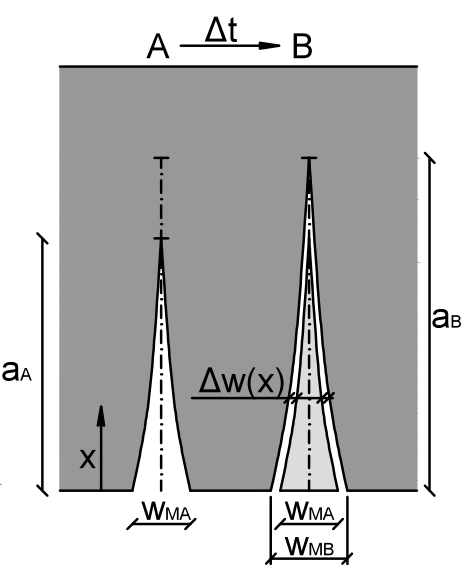

Figure 7: Local crack opening rate.

Once $K_{I c}^{i n i}$ has been determined, which is considered to have a low variation under a change of loading rate and, so, can be kept constant, the new value of $K_{I c}^{u n}$ is obtained by adding the rate-affected $K_{I c}^{c}$ to $K_{I c}^{\text {ini }}$. With this methodology, it is possible to easily evaluate the rate effect in the double- $K$ parameters.

Furthermore, although $K_{I c}^{c}$ was initially established to calculate the difference between $K_{I c}^{i n i}$ and $K_{I c}^{u n}$, it is possible to obtain this parameter at every point of the $P-w_{M}$ curve, each with a corresponding equivalent elastic crack length. Thus, $K_{I c}^{i n i}+K_{I c}^{c}$ at each point generates a toughness-crack length curve $\left(K_{I c^{-}}\right.$ $a$ ), with an equivalent resistance curve $R-a$ through Eq. 7:

$$
R=\frac{K_{I c}^{2}}{G_{F}}
$$

This methodology aims to get a term $\left(K_{I C}^{c}\right)$ capable to relate the corresponding $K_{I c}^{u n}$ and $K_{I c}^{i n i}$ values whatever the loading velocity, 
consideration that the original method did not include. Moreover, by assuming the hypothesis that $K_{I c}^{i n i}$ is less sensitive to rate effects than $K_{I c}^{u n}$ and, so, it will experience little change regardless the loading velocity, $K_{I c}^{c}$ would help to estimate $K_{I c}^{u n}$ for a certain loading rate starting from a known $K_{I c}^{i n i}$ value measured with quasi-static tests. Inversely, if what is known is a $K_{I c}^{u n}$ measured in a case with a relevant loading velocity, a $K_{I c}^{c}$ calculated accordingly could serve to detract the rate effect from that parameter to obtain a reference $K_{I c}^{i n i}$ and, after that, adding a new $K_{I c}^{c}$ without rate influence would provide the static $K_{I c}^{u n}$ that corresponds to that concrete.

Double- $K$ curves were also calculated in the previous work in static conditions [13]. Now, they are also presented in comparison with the effect of the loading rate.

\section{RESULTS AND DISCUSSION}

The first results to analyse consist in the double- $K$ parameters that can be measured directly on the static and rate-affected $P-w_{M}$ curves, respectively. Figure 8 shows that, while $K_{I c}^{\text {un }}$ experiences an increment when considering the loading velocity, $K_{I c}^{\text {ini }}$ keeps around a constant value. This fact supports the idea presented above, which considers that a static $K_{I c}^{i n i}$ may not differ much from its corresponding rate-affected value and, so, it can be taken as the base for reaching $K_{I c}^{u n}$ considering the load velocity by means of $K_{I c}^{c}$.

The similarity between both $K_{I c}^{\text {ini }}$ values is partially explained because the numerical model does not consider the variation of the elastic modulus $E$ due to rate effects. However, this would not induce an appreciable change in $K_{I C}^{i n i}$ and, thus, the approximation is correct.

Next, $K_{I c}^{c}$ parameters are introduced. On the one hand, the cohesive toughness calculated from the rate-affected $P-w_{M}$ curves, with the appropriate viscous parameters, is detracted from its corresponding $K_{I c}^{u n}$ to obtain $K_{I c}^{i n i}$. As shown in Fig. 8, this new $K_{I c}^{i n i}$ value coincides with the one already measured up to a beam size of $600 \mathrm{~mm}$, although later it continues growing following the increasing tendency of $K_{I c}^{u n}$. Then, for typical specimen sizes in laboratory, the $K_{I c}^{c}$ parameter with a viscous factor works as well as its static counterpart for estimating $K_{I c}^{i n i}$ (Fig. 6).

On the other hand, the modified $K_{I c}^{c}$, obtained now from the static $P-w_{M}$ curves, is added to the static $K_{I c}^{i n i}$. This new $K_{I c}^{u n}$ is, again, a good approximation for the one that can be directly measured with the rate-affected curves until a size of $600 \mathrm{~mm}$, remaining, in any case, within a $20 \%$ of variation on the safe side for larger sizes.

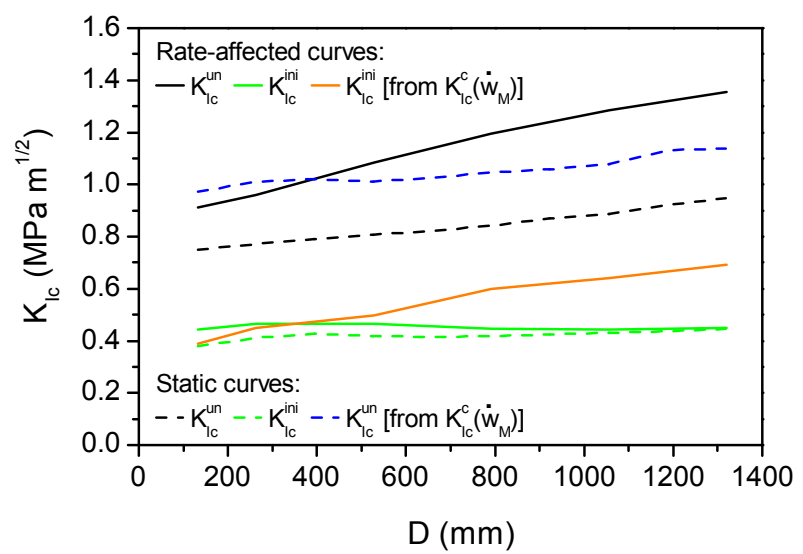

Figure 8: Static and rate-affected double- $K$ parameters.

Focusing on these two values of the cohesive toughness, with the same viscous parameters and crack opening rates, Fig. 9 presents a direct comparison between them, without the distortion introduced in Fig. 8 by the size effect of $K_{I c}^{u n}$. This way, it is possible to observe that both $K_{I c}^{c}$ parameters are very similar in the entire size range, only slightly different for smaller sizes.

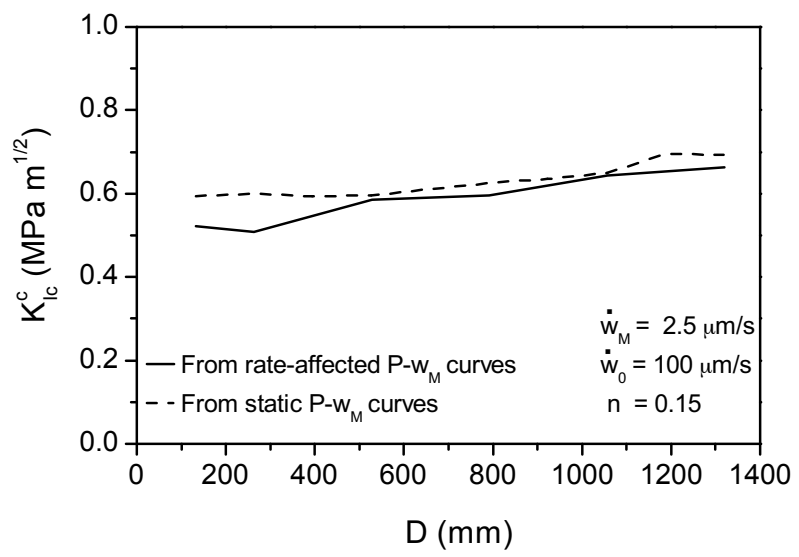

Figure 9: Comparison of $K_{I c}^{c}$ calculated from static and rate-affect $P-w_{M}$ curves, applying the viscous factor in both cases. 
The value of $K_{I c}^{c}$ with a viscous factor for a certain point depends on the distribution of the crack width along its length in the propagation state of the previous point on the curve. Therefore, the likeness between both groups of $K_{I C}^{c}$ means that there exists a relation between consecutive $P-w_{M}$ points that is not affected by rate effects. This a relevant result as it validates the hypothesis that rate effects can be estimated from static $P-w_{M}$ curves within a range of tolerance, and vice versa. Experimental tests with different specimen sizes and with high loading rates are required to contrast this observation.

In the following, rate effects on the double$K$ method adapted with this methodology are studied through $K_{I C}^{c}$ obtained with the static $P_{-}$ $w_{M}$ curves, depending on the variables that affect Eq. 1. Parametric analyses have been performed on the $n$ parameter of the viscous factor, the crack mouth opening rate for a constant value for any beam size and the crack mouth opening rate for a value proportional to the beam size.

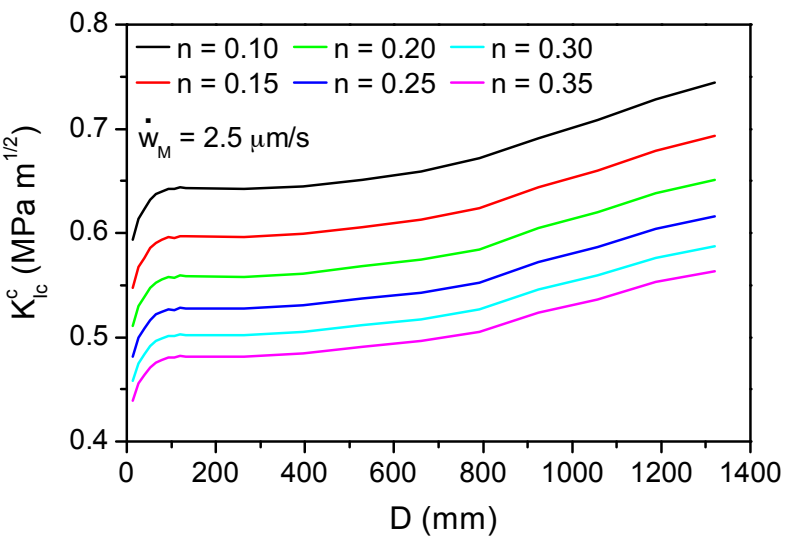

Figure 10: Rate effect varying $n$.

In Fig. 10, $K_{I c}^{c}$ diminishes for higher $n$. This is in consonance with [7], where an increasing value of $n$ obtains decreasing values of load up to a certain displacement rate/reference opening rate relation. From that point on, the effect is the opposite. Then, the relation of $\dot{w}_{0}=100 \mu \mathrm{m} / \mathrm{s}$ and $\dot{w}_{M}=2.5 \mu \mathrm{m} / \mathrm{s}$ is to the left of that inflection point.

Fig. 11 and 12 show the variation of $K_{I C}^{c}$ depending on $\dot{w}_{M}$, keeping $n=0.15$. In the first case, $\dot{w}_{M}$ is constant for every specimen, analysing different velocities. In the second case, $\dot{w}_{M}$ is proportional to the beam size, considering only a starting value of $2.5 \mu \mathrm{m} / \mathrm{s}$, corresponding to the smallest size $\left(0.1 l_{c h}\right)$.

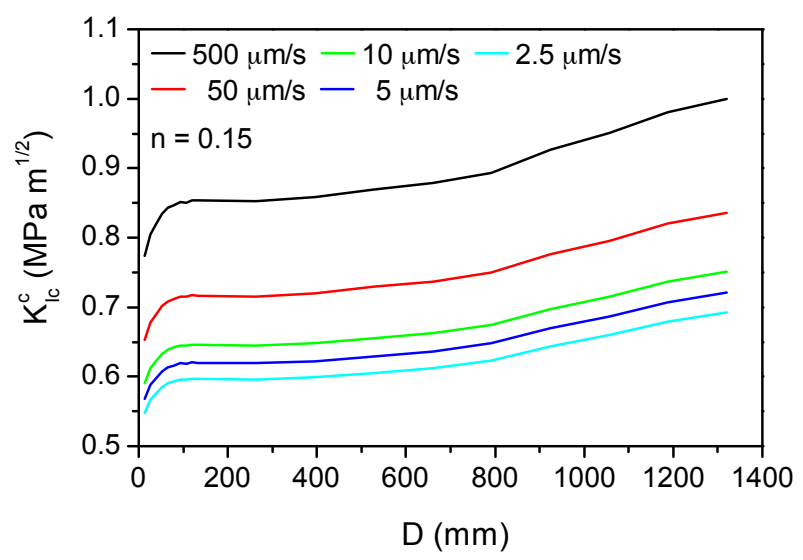

Figure 11: Rate effect varying $\dot{w}_{M}$, constant for all sizes.

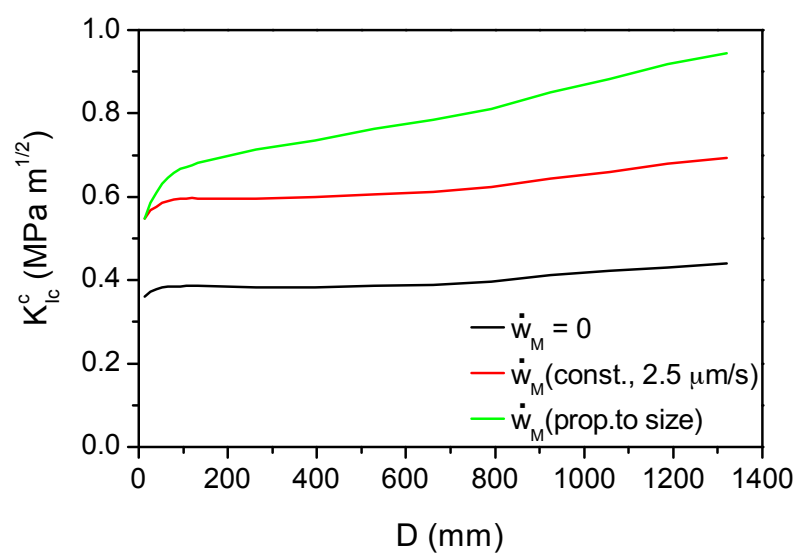

Figure 12: Rate effect varying $\dot{w}_{M}$, size dependent.

For constant opening rates, each $K_{I c}^{c}$ curve presents a certain size effect, increasing for larger sizes. The different $\dot{w}_{M}$ values form a family of parallel curves. The interval between them is proportionally bigger for smaller opening rates than for larger ones. An increase of $\dot{w}_{M}$ results in an increase of the $K_{I c}^{c}$ value, although not in the same magnitude, as from $2.5 \mu \mathrm{m} / \mathrm{s}$ to $500 \mu \mathrm{m} / \mathrm{s}$ the variation of $K_{I c}^{c}$ is about a 40\%. Figure 13 shows the increase tendency of $K_{I c}^{c}$, depending on the crack mouth opening rate for the beam of size $D=1 l_{c h}$. The relation is linear for a logarithmic horizontal scale, which means that for an exponential increase of $\dot{w}_{M}$, the variation in $K_{I C}^{c}$ is moderate in relative terms.

Figure 12 also shows the static $K_{I c}^{c}$ curve and repeats the corresponding one for 
$\dot{w}_{M}=2.5 \mu \mathrm{m} / \mathrm{s}$. Again, their trajectories are parallel, with the rate-affected curve a $50 \%$ higher. In the case where the opening rate increases with the size, the size effect in $K_{I c}^{c}$ is amplified, from that $50 \%$ of increase for the smallest size to a $100 \%$ for the largest one $\left(10 l_{c h}\right)$.

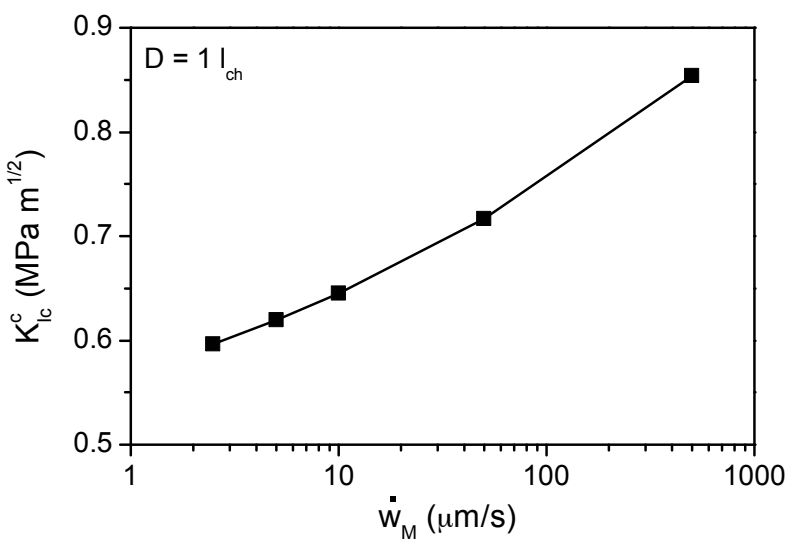

Figure 13: Variation of $K_{I c}^{c}$ with $\dot{w}_{M}$.

Finally, the rate effect in $K_{I c}^{c}$ is also presented in the form of $R$-like curves, studying the evolution of the cohesive toughness along the crack for different crack opening rates. Figure 14 presents the results for $D=1 l_{c h}$ in non-dimensional units and in terms of $K_{I c}^{c}$. As $K_{I c}^{i n i}$ is a constant, $K_{I c}^{c}$ curves have the same shape than $K_{I c}\left(K_{I c}^{i n i}+K_{I c}^{c}\right)$. By Eq. 7, it is possible to convert this data into an $R$ curve. These curves follow the same tendency already observed in Fig. 11, with a higher value of $K_{I c}^{c}$ for larger velocities. Besides, it is possible to appreciate an increasing tendency along the crack, although very slight, so it can be neglected.

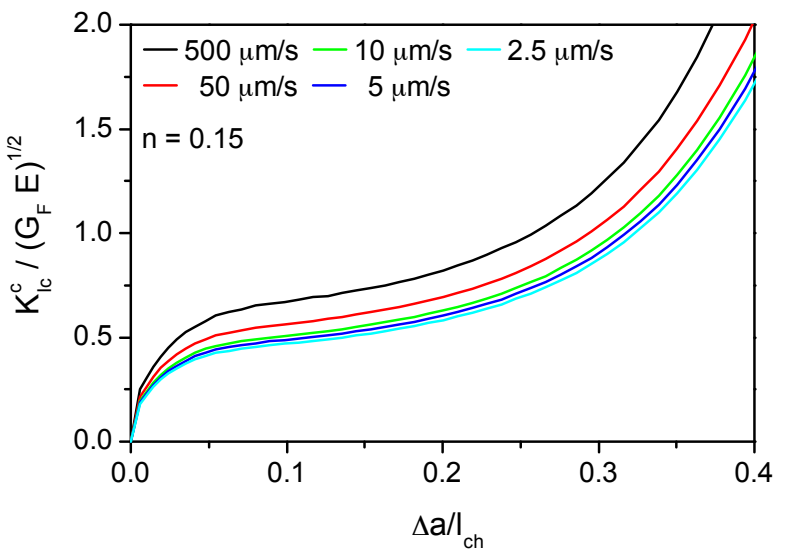

Figure 14: Cohesive toughness evolution along the crack for different crack opening rates.
This graph is useful to predict the behaviour of a concrete during a test where the opening rate is changed (intentionally or not), for example, in order to reduce its duration. If this has been a sudden change, the trajectory followed on a $K_{I c}^{c}-\Delta a$ curve would suffer a jump to another one, continuing along this new curve after that.

\section{CONCLUSIONS}

In the present work, a viscous factor has been introduced in the double- $K$ method in order to reproduce the loading rate influence. $K_{I c}^{c}$ is the parameter that integrates the cohesive toughness developed along the FPZ of the crack at a certain loading state. With its help, it is possible to modify the associated stress for a given crack opening and, so, include the increase in the response of concrete against fracture that is empirically observed.

With the simple approach presented here, it has been possible to reproduce in the double- $K$ parameters typical increments due to rate effects in other fracture parameters. This study shows how relatively small crack opening rates produce a clear increase in the result of toughness parameters, with a damped growing tendency.

The election of the parameters of the viscous factor has also an important role as they control the final value of $K_{I c}^{c}$ and, therefore, they must be properly chosen for the material under study.

The proposed methodology can also determine the resistance curves for a given specimen for various crack opening velocities, which allows following the evolution during a fracture process where changes of the loading rate take place.

\section{ACKNOWLEDGEMENTS}

The authors acknowledge the financial support to perform this research from the Ministerio de Economía y Competitividad, Spain, under grant number BIA2015-68678C2-1-R, and from the Junta de Comunidades de Castilla-La Mancha, under grant number PEII-2014-016-P and a predoctoral grant. 


\section{REFERENCES}

[1] Xu, S. L. and Reinhardt, H. W. 1999a. Determination of double-K criterion for crack propagation in quasi-brittle materials, Part I: Experimental investigation of crack propagation. International Journal of Fracture 98:111-149.

[2] $\mathrm{Xu}, \mathrm{S} . \mathrm{L}$. and Reinhardt, H. W. 1999b. Determination of double-K criterion for crack propagation in quasi-brittle materials, Part II: Analytical evaluating and practical measuring methods for three-point bending notched beams. International Journal of Fracture 98:151-177.

[3] $\mathrm{Xu}, \mathrm{S}$. L. and Reinhardt, H. W. 1999c. Determination of double-K criterion for crack propagation in quasi-brittle materials, Part III: Compact tension specimens and wedge splitting specimens. International Journal of Fracture 98:179-193.

[4] Xu, S. L. and Reinhardt, H. W. 2000. A simplified method for determining double-K fracture parameters for three-point bending tests. International Journal of Fracture 104(2):181-209.

[5] DL/T 5332-2005. 2006. Norm for Fracture Test of Hydraulic Concrete [S]. Power Press, Beijing, China.

[6] Bažant, Z. P. and Jirásek, M. 1993. R-curve modelling of rate and size effects in quasibrittle fracture. International Journal of Fracture 62:355-373.
[7] Rosa, A. L., Yu, R. C., Ruiz, G., Saucedo, L. and Sousa, J. L. A. O. 2012. A loading rate dependent cohesive model for concrete fracture. Engineering Fracture Mechanics 82:195-208.

[8] Planas, J. and Elices, M. 1991. Fracture criteria for concrete: mathematical approximations and experimental validation. Engineering Fracture Mechanics 35:87-94.

[9] Petersson, P.E. 1981. Crack growth and development of fracture zone in plain concrete and similar materials. Report No. TVBM100. Lund Institute of Technology.

[10] Guinea, G.V., Planas, J. and Elices, M. 1994. A general bilinear fit for the softening curve of concrete. Materials and Structures 27:99105.

[11] Ruiz, G. 2001. Propagation of a cohesive crack crossing a reinforcement layer. International Journal of Fracture 111:265282.

[12] Tada, H., Paris, P.C., Irwin, G.R. 1985. The Stress Analysis of Cracks Handbook, Paris Productions Incorporated, St. Louis, Missouri, USA.

[13] Ruiz, G., Ortega, J. J. and Yu, R. C. 2015. Size effect on double-K fracture parameters in concrete. Anales de Mecánica de la Fractura 32:184-189.

[14] Reinhardt, H. W., Cornelissen, H. A. W. and Hordijk, D. A. 1986. Tensile tests and failure analysis of concrete. Journal of Structural Engineering 112(11):2462-2477. 